\title{
Lipocalina asociada con gelatinasa del neutrófilo como predictor de disfunción del injerto a un año del trasplante renal
}

\author{
Lina María Serna-Higuita, ${ }^{1,2}$ John Fredy Nieto-Ríos, ${ }^{2}$ Catalina Ocampo-Kohn, ${ }^{2}$ Arbey Aristizabal-Alzate, ${ }^{2}$ \\ Gustavo Adolfo Zuluaga-Valencia² y Fabián Jaimes-Barragán ${ }^{3}$ \\ ${ }^{1}$ Hospital Universitario de Tübingen, Departamento de Epidemiología y Biometría, Tübingen, Alemania; ${ }^{2}$ Universidad de Antioquia, Hospital Pablo \\ Tobón Uribe, Departamento de Nefrología y Trasplante Renal, Medellín, Colombia; ${ }^{3}$ Universidad de Antioquia, Hospital Pablo Tobón Uribe, \\ Departamento de Investigación, Medellín, Colombia
}

\section{Resumen}

Introducción: Estudios recientes sugieren que la lipocalina asociada con la gelatinasa del neutrófilo urinaria (NGALu) es superior a la creatinina para la detección temprana de la disfunción del injerto renal, pero son pocos los estudios que evalúan su utilidad como predictor a largo plazo de dicha función. Objetivo: Explorar si los valores de NGALu en las primeras 48 horas después del trasplante renal predicen la función del injerto a largo plazo. Método: Cohorte prospectiva en la que se evaluaron los valores de NGALu a las 2, 12, 24 y 48 horas postrasplante renal. Resultados: Se valoraron 79 pacientes trasplantados renales. Al año de seguimiento, 30.4 de los pacientes presentó disfunción del injerto. No se encontraron diferencias estadísticamente significativas entre los valores de NGALu y la función a un año del injerto renal ( $p=0.65)$; el análisis multivariado mostró que ningún valor de NGALu fue un marcador predictor de disfunción del injerto a un año del trasplante renal. Conclusión: Los valores de NGALu obtenidos en las primeras 48 horas postrasplante no se asociaron con disfunción del injerto a un año del trasplante renal.

PALABRAS CLAVE: Lipocalina. Pérdida del injerto. Trasplante renal.

\begin{abstract}
Introduction: Recent studies suggest that urinary neutrophil gelatinase-associated lipocalin (UNGAL) is superior to creatinine for renal graft dysfunction early detection, but there are only few studies assessing its usefulness as long-term predictor of said function. Objective: To explore if UNGAL values within the first 48 hours after kidney transplantation predict graft function on the long term. Method: Prospective cohort, where UNGAL values were assessed at 2, 12, 24 and 48 hours post-kidney transplantation. Results: Seventy-nine kidney transplant recipients were evaluated. At one year of follow-up, $30.4 \%$ of patients had graft dysfunction. No statistically significant differences were found between the UNGAL values and the renal graft function at one year $(p=0.65)$; the multivariate analysis showed that no UNGAL value was a predictor marker of graft dysfunction at one year of kidney transplantation. Conclusion: The UNGAL values obtained within the first 48 hours post-transplant were not associated with graft dysfunction at one year of kidney transplantation.
\end{abstract}

KEY WORDS: Lipocalin. Graft loss. Kidney transplantation.

\section{Introducción}

La función retardada del injerto renal (FRI), definida como la necesidad de terapia de reemplazo renal durante los primeros siete días del trasplante o el no descenso de la creatinina sérica $<10 \%$ durante tres días consecutivo, ${ }^{1}$ es una de las complicaciones más comunes después del trasplante renal; ${ }^{2}$ se origina por
Correspondencia: Lina María Serna-Higuita E-mail: Im.serna@ hotmail.com
Fecha de recepción: 25-11-2016

Fecha de aceptación: 05-06-2017 DOI://dx.doi.org/10.24875/GMM.18003025
Gac Med Mex. 2018;154:275-282

Disponible en PubMed www.gacetamedicademexico.com 
la lesión isquemia-reperfusión que se presenta después del trasplante renal y ha demostrado ser un factor de riesgo para rechazo agudo y nefropatía crónica del injerto renal, que puede favorecer la pérdida de este en el primer año. ${ }^{3-6}$ Se han explorado diferentes estrategias terapéuticas para evitar la FRI, sin embargo, las opciones exitosas en modelos animales no han sido reproducidas en humanos ${ }^{3,7}$ debido a la identificación tardía de esta lesión,,$^{3,8-10}$ ya que los biomarcadores actuales para su diagnóstico (que incluyen la creatinina sérica y la diuresis) son poco sensibles para predecirla..$^{11-14}$

En la actualidad existe la necesidad de marcadores con mayor sensibilidad y especificidad que permitan un diagnóstico temprano de la FRI y de la lesión renal subclínica, ${ }^{15}$ con el objetivo de establecer intervenciones terapéuticas que incrementen la supervivencia del injerto renal..$^{3,16}$ Estudios recientes sugieren que el NGAL urinario (NGALu) (urinary neutrophil gelatinase-associated lipocalin) podría ser superior a la creatinina sérica para la detección temprana de la FRI, ya que su elevación puede suceder en las primeras 12 horas después del trasplante renal en pacientes con disfunción subclínica, ${ }^{3,5,7,76-26}$ sin embargo, son pocos los estudios donde se ha evaluado la utilidad del NGALu como predictor a largo plazo de la función del injerto renal y no es claro si sus valores pueden predecir disfunción del mismo. ${ }^{2,18,21,27,28}$

En el Hospital Pablo Tobón Uribe, Antioquia, Medellín, Colombia, predominan los trasplantes de donante fallecido, en los cuales la frecuencia de FRI es mayor $y$, por lo tanto, es de esperar mayor frecuencia de lesión renal subclínica. Consideramos, que el NGALu podría ser un buen marcador pronóstico para anticipar la función renal a un año del trasplante renal. En un estudio realizado previamente por nuestro grupo se encontró que el valor de NGALu 48 horas después del trasplante renal fue un predictor temprano de FRI, sin embargo, no resultó ser mejor predictor al ser comparado con el de la creatinina sérica. ${ }^{29}$

En este artículo describimos los resultados en una cohorte de pacientes seguida por un año, con el objetivo de explorar si los valores de NGALu en las primeras 48 horas después del trasplante renal predicen la función del injerto a largo plazo.18

\section{Método}

Estudio tipo cohorte prospectiva. La cohorte se estableció inicialmente para evaluar la relación entre los valores del NGALu obtenidos a las horas 1, 12, 24 y
48 después del trasplante renal y la presencia o no de FRl;29 posteriormente para la realización de este segundo estudio se continuó el seguimiento de la cohorte por un periodo de un año posterior al trasplante renal.

Este estudio fue realizado en el Hospital Pablo Tobón Uribe; se incluyeron los pacientes mayores de 12 años con diagnóstico de enfermedad renal crónica (ERC) terminal que recibieron trasplante renal entre noviembre de 2012 y diciembre de 2013. Se excluyeron los pacientes con anuria en la primera hora después del trasplante renal. El tratamiento de inducción utilizado en esta cohorte consistió en $20 \mathrm{mg}$ de basiliximab los días 0 y 4 en pacientes con bajo riesgo inmunológico $01 \mathrm{mg} / \mathrm{k} /$ día de timoglobulina por tres a cinco días en pacientes con alto riesgo inmunológico y tres bolos de metilprednisolona. El tratamiento de mantenimiento consistió en un triple esquema de inmunosupresión con prednisolona, un antiprofilerativo (micofenolato o azatriopina) y un inhibidor de calcineurina (tacrolimus o ciclosporina). Todos los pacientes fueron seguidos durante el año siguiente al trasplante renal por nefrólogos que cegados a los valores del NGALu.

El desenlace primario se definió como una tasa de filtración glomerular (TFG) $<60 \mathrm{~mL} /$ minuto/1.73 $\mathrm{m}^{2}$ de superficie corporal (sc), estimada por la ecuación CKD-EP ${ }^{30}$ durante el primer año después del trasplante renal. Los valores de creatinina sérica para la anterior estimación fueron medidos durante el seguimiento mensual de los pacientes en el primer año postrasplante renal. Como variable de exposición se utilizaron los valores del NGALu obtenidos a las horas 1, 12, 24 y 48 posteriores al trasplante renal. Otros parámetros clínicos evaluados fueron sexo, edad, etiología de la enfermedad renal crónica (ERC), antecedentes de trasplante renal y de diálisis antes del trasplante, tiempo de isquemia fría y caliente, tratamiento inmunosupresor y presencia de FRI; esta última fue definida por la necesidad de diálisis en la primera semana después del trasplante renal o disminución de la creatinina sérica $<10 \%$ durante tres días consecutivos 0 un valor de creatinina sérica $>3 \mathrm{mg} / \mathrm{dL}$ al quinto día del trasplante renal.

\section{Fuente de los datos, procedimientos de laboratorio}

Se recolectaron $10 \mathrm{~mL}$ de orina en cada paciente directamente de la bolsa unida a la sonda vesical permanente de los primeros días postrasplante; antes 
se desechó la orina de la bolsa para recolectar orina fresca. Las muestras se tomaron a las horas 1, 12, 24 y 48 postrasplante y antes de una hora de la recolección se enviaron al laboratorio del hospital, donde se centrifugaron a 400 revoluciones por minuto y se almacenaron a $-70{ }^{\circ} \mathrm{C}$ hasta ser procesadas. La determinación del NGALu se realizó mediante inmunoensayo turbidimétrico, del fabricante Sentinel Diagnostics (REF 1P9300) sobre la plataforma Architect $^{\circledR}$ i8000 de Abbott. Las muestras fueron procesadas en un periodo < 3 meses desde el momento de la toma. La creatinina sérica se midió en forma rutinaria por método enzimático, se tomó diariamente durante la primera semana del trasplante y posteriormente en forma mensual en los siguientes 12 meses.

\section{Tamaño de muestra y poder}

Se utilizó un modelo de tiempo hasta el evento, considerando los valores del NGALu en las primeras 48 horas del trasplante como la variable de exposición y la $\mathrm{TFG}<60 \mathrm{~mL} /$ minuto $/ 1.73 \mathrm{~m}^{2} \mathrm{sc}$ en el primer año postrasplante como el desenlace de interés. ${ }^{31}$

Dado que se analizó una cohorte con un tamaño de muestra definido (79 pacientes), se estimó el poder para detectar la razón de riesgo (RR) de disfunción del injerto de acuerdo con los valores de NGALu en las primeras 48 horas postrasplante. Los supuestos utilizados fueron: RR de $2,{ }^{29,32,33}$ proporción esperada de pacientes trasplantados con disfunción del injerto de $35 \% .^{10,22}$ De acuerdo con los anteriores supuestos y con un error alfa de 0.05 , esta cohorte tuvo un poder de 0.881 para identificar un RR de 2 ante TFG $<60 \mathrm{~mL} /$ minuto/1.73 $\mathrm{m}^{2} \mathrm{sc}$ durante el primer año postrasplante en los pacientes con valores de NGALu elevados.

Los pacientes fueron agrupados de acuerdo con el desenlace primario: presencia o no de disfunción del injerto a un año del trasplante renal; en ambos grupos se exploró si los valores de NGALu predecían disfunción del injerto renal.

\section{Análisis estadístico}

Se utilizó estadística descriptiva y se verificó la distribución de las variables por medio de la prueba Kolmogorov-Smirnov. Las variables cuantitativas se presentaron en medias y desviación estándar o medianas y rangos intercuartílicos, según la distribución de los datos; las variables cualitativas se analizaron como proporciones. Se realizaron diagramas de dispersión entre los valores de NGALu en las primeras 48 horas y la TFG por CKD-EPI a un año del trasplante renal; posteriormente se evaluó la correlación entre estas dos variables continuas utilizando el coeficiente de correlación de Pearson ( $r$ ).

Se exploró la capacidad discriminativa de los diferentes valores del NGALu para la detección de disfunción del injerto renal durante el primer año del trasplante y se realizó un análisis de supervivencia de Kaplan-Meier. También se realizó un modelo de riesgos proporcionales de Cox.

\section{Aspectos éticos}

Este estudio fue aprobado por el comité de ética e investigación del Hospital Pablo Tobón Uribe y se siguieron las normas sobre aspectos éticos de la investigación en seres humanos contenidas en la resolución 008430 de 1993, emitida por el Ministerio de Salud de Colombia; además, se conservó la confidencialidad de los datos personales de los pacientes incluidos. Por otro lado, este estudio no representó riesgos para los pacientes, ya que los exámenes en sangre formaban parte del protocolo de trasplante renal y las muestras del NGALu fueron tomadas directamente de la bolsa de recolección unida a la sonda vesical permanente que tenían los pacientes después del trasplante renal.

\section{Resultados}

Entre noviembre de 2012 y diciembre de 2013 se realizaron 83 trasplantes renales en el Hospital Pablo Tobón Uribe; dos pacientes fueron excluidos por anuria en las primeras horas y dos por ser menores de 12 años, quedando 79 pacientes. Durante el seguimiento un paciente murió, sin embargo, su TFG fue normal al momento de la muerte, por lo que se incluyó en el grupo de pacientes. Todos los pacientes recibieron un trasplante renal de donante fallecido, pero ninguno que incluyera alguno de los criterios extendidos (donante $>60$ años o entre 50 y 60 años con al menos dos de los siguientes criterios: historia de hipertensión arterial, niveles de creatinina plasmática $>1.5 \mathrm{mg} / \mathrm{dL}$ o con accidente cerebrovascular como causa del fallecimiento). La mayoría de los pacientes ( $n=44,55.7 \%$ ) fue del sexo masculino, la edad media fue de $39.6 \pm 4.18$ años; para $91.1 \%(n=72)$ se trataba de su primer trasplante y $82.3 \%(n=65)$ estaba en diálisis antes del trasplante, con una mediana de 23.5 meses (rango intercuartílico $[R \mid Q]=4-48)$. El $51.9 \%(n=41)$ recibió 
Gaceta Médica de México. 2018;154

Tabla 1. Análisis descriptivo agrupado por el desenlace disfunción del injerto renal durante el primer año postrasplante

\begin{tabular}{|c|c|c|c|c|}
\hline \multirow[t]{2}{*}{ Variable } & \multicolumn{2}{|c|}{$\mathrm{TFG} \leq 60 \mathrm{~mL} /$ minuto $(\mathrm{n}=24)$} & \multicolumn{2}{|c|}{$\begin{array}{c}\mathrm{TFG}>60 \mathrm{~mL} / \text { minuto } \\
(\mathrm{n}=55)\end{array}$} \\
\hline & $\mathrm{n}$ & $\%$ & $\mathbf{n}$ & $\%$ \\
\hline Sexo masculino & 8 & 33.3 & 36 & 65.5 \\
\hline Antecedentes de transfusiones previas & 19 & 79.2 & 46 & 83.6 \\
\hline Segundo trasplante & 0 & 0 & 7 & 12.7 \\
\hline Diálisis previa & 19 & 79.2 & 46 & 83.6 \\
\hline Inducción con basiliximab & 5 & 20.8 & 20 & 36.4 \\
\hline Inducción con timoglobulina & 19 & 79.2 & 35 & 63.5 \\
\hline Función retardada del injerto renal & 4 & 16.7 & 9 & 16.4 \\
\hline Necesidad de diálisis en la primera semana del trasplante renal & 1 & 4.2 & 4 & 7.3 \\
\hline \multirow[t]{2}{*}{ Rechazo agudo en el primer año del trasplante } & 10 & 41.7 & 6 & 10.9 \\
\hline & \multicolumn{2}{|c|}{ Media $\pm \mathrm{DE}$} & \multicolumn{2}{|c|}{ Media \pm DE } \\
\hline Edad al trasplante (años) & \multicolumn{2}{|c|}{$40.67 \pm 15.07$} & \multicolumn{2}{|c|}{$39.07 \pm 13.88$} \\
\hline Edad del donante (años) & \multicolumn{2}{|c|}{$35.67 \pm 15.78$} & \multicolumn{2}{|c|}{$27 \pm 11.28$} \\
\hline Creatinina del donante $(\mathrm{mg} / \mathrm{dL})$ & \multicolumn{2}{|c|}{$0.98 \pm 0.34$} & \multicolumn{2}{|c|}{$0.84 \pm 0.27$} \\
\hline Tiempo de isquemia fría (horas) & \multicolumn{2}{|c|}{$14.54 \pm 6.08$} & \multicolumn{2}{|c|}{$15.13 \pm 5.80$} \\
\hline Tiempo de isquemia caliente (minutos) & \multicolumn{2}{|c|}{$33.75 \pm 7.11$} & \multicolumn{2}{|c|}{$32.81 \pm 7.51$} \\
\hline Incompatibilidad DR & \multicolumn{2}{|c|}{$1.5 \pm 0.5$} & \multicolumn{2}{|c|}{$1.2 \pm 0.6$} \\
\hline Incompatibilidad total HLA & \multicolumn{2}{|c|}{$4.9 \pm 0.8$} & \multicolumn{2}{|c|}{$4.2 \pm 1$} \\
\hline \multicolumn{5}{|l|}{ TFG postrasplante renal (mL/minuto/1.73 $\left.\mathrm{m}^{2} \mathrm{sc}\right)$} \\
\hline $\begin{array}{l}\text { A los } 5 \text { días } \\
\text { A los } 6 \text { meses }\end{array}$ & \multicolumn{2}{|c|}{$\begin{array}{l}56.93 \pm 26.62 \\
53.37 \pm 24.25\end{array}$} & \multicolumn{2}{|c|}{$\begin{array}{l}58.59 \pm 32.52 \\
68.22 \pm 19.56\end{array}$} \\
\hline & \multicolumn{2}{|c|}{ Mediana (RIQ) } & \multicolumn{2}{|c|}{ Mediana (RIQ) } \\
\hline \multicolumn{5}{|l|}{ Creatinina postrasplante renal (mg/dL) } \\
\hline Día 1 & \multicolumn{2}{|c|}{$7.73(5.4-13.6)$} & \multicolumn{2}{|c|}{$8.8(6.6-12.6)$} \\
\hline Día 2 & \multicolumn{2}{|c|}{$5.16(3.34-6.41)$} & \multicolumn{2}{|c|}{$6.1(3.32-8.79)$} \\
\hline Día 3 & & $-2.22)$ & & $2.59)$ \\
\hline Día 4 & & $-1.89)$ & & $-1.9)$ \\
\hline Día 5 & & $-1.77)$ & & $-1.68)$ \\
\hline Día 30 & & 2.16) & & $-1.52)$ \\
\hline NGALu postrasplante renal (ng/mL) & & & & \\
\hline A la hora $(n=79)$ & & 1180.4) & & 1203) \\
\hline A las 12 horas $(n=78)^{*}$ & & $-546.5)$ & & $-686.9)$ \\
\hline A las 24 horas $(n=78)^{*}$ & & $-494.3)$ & & 446.3) \\
\hline A las 48 horas $(n=77)$ & & $-189.6)$ & & 15.8) \\
\hline
\end{tabular}

TFG=tasa de filtración glomerular, $\mathrm{RIQ}=$ rango intercuartílico.

*Una paciente presentó trombosis y pérdida del injerto renal posterior a la primera hora del trasplante, por lo cual solo fue posible la toma de la primera muestra del NGALu.

transfusiones previas y el tiempo de isquemia fría tuvo una media de $15.04 \pm 5.84$ horas. La FRI se presentó en 13 pacientes (16.5\%), cinco de ellos $(6.3 \%)$ necesitaron diálisis durante la primera semana. Al año de seguimiento, 24 pacientes (30.4\%) presentaron disfunción del injerto renal; las características demográficas agrupadas por presencia o no de disfunción del injerto renal se encuentran en la Tabla 1.

No se encontró correlación entre la TFG por CKD-EPI a un año del trasplante y los valores del NGALu a las horas 1, 12, 24 y $48(r=0.14, p=0.2 ; r=0.05, p=0.66$; $r=0.06, p=0.61 ; r=0.11 p=0.34$, respectivamente); 


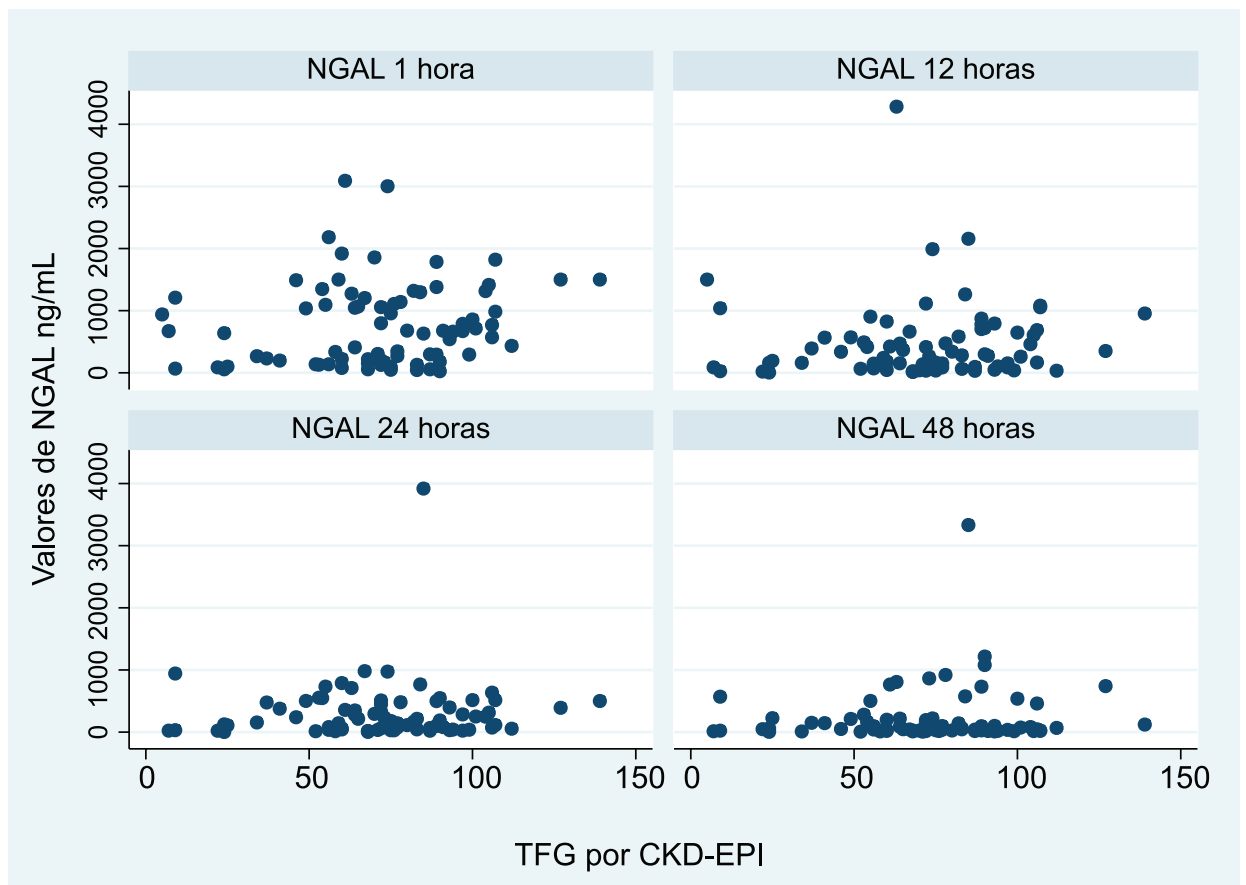

Figura 1. Diagramas de dispersión de los valores de NGALu en las primeras 48 horas postrasplante y los valores de la tasa de filtración glomerular estimada por la fórmula CKD-EPI al año del trasplante renal.

tampoco se observó otro patrón de comportamiento (Figura 1).

La Figura 2 muestra las curvas ROC y sus respectivas áreas bajo la curva por cada valor de NGALu a las horas $1,12,24$ y 48 postrasplante renal con respecto al desenlace disfunción del injerto renal al año (0.569 [IC $95 \%=0.424-0.715$ ], 0.542 [IC $95 \%=0.406-0.678]$, $0.55[$ IC $95 \%=0.398-0.702]$ y $0.55[$ [C $95 \%=0.408-0.692]$, respectivamente.

\section{Comportamiento de los valores de NGALU}

El $75 \%$ de los pacientes $(n=59)$ tuvo descenso del NGALu superior a $50 \%$ en las primeras 48 horas, $15 \%(n=12)$ tuvo un descenso $\leq 50 \%$ y en ocho pacientes (10\%) aumentaron los valores del NGALu respecto a la primera medición. El porcentaje de pacientes libres de disfunción del injerto renal a los seis y 12 meses postrasplante según el comportamiento de los valores de NGALu fue de 88 y $71 \%$ en el grupo con descenso > $50 \%$; 74 y $65 \%$ en el grupo con descenso $<50 \%$, y 62 y $62 \%$, respectivamente, en el grupo en el que los valores de NGALu aumentaron (Figura 3) (prueba log rank $p=0.65$ ).

El modelo de riesgos proporcionales de COX según el comportamiento del NGALu 48 horas postrasplante no mostró asociación estadísticamente significativa con la ocurrencia de disfunción del injerto renal a un año del trasplante (Tabla 2).

En los pacientes con disfunción del injerto $(n=24)$, $16.7 \%(n=4)$ había presentado previamente FRI, comparado con $16.4 \%(9 / 54)$ en pacientes con TFG $>60 \mathrm{~mL} /$ minuto $/ 1.73 \mathrm{~m}^{2} \mathrm{sc}(\mathrm{p}=0.6)$.

\section{Discusión}

En esta cohorte de 79 pacientes con trasplante renal, todos de donante fallecidos, no se demostró que los valores del NGALu en las primeras 48 horas postrasplante fueran predictores de una insuficiente función del injerto a 12 meses de seguimiento. Tampoco se encontró correlación entre los valores del NGALu y la TFG a un año del trasplante renal ni se logró establecer un punto de corte para el NGALu que lograra discriminar a los pacientes con disfunción del injerto.

En la actualidad no existe un biomarcador que permita predecir en forma temprana el resultado a largo plazo del injerto renal. El NGALu ha sido propuesto como un biomarcador útil para la detección temprana de FRI, ${ }^{19,28,34-36}$ sin embargo, son pocos los estudios en los que se ha evaluado su papel como un biomarcador a largo plazo de la disfunción del injerto renal. ${ }^{37}$ Consideramos útil explorar si los valores del NGALu 


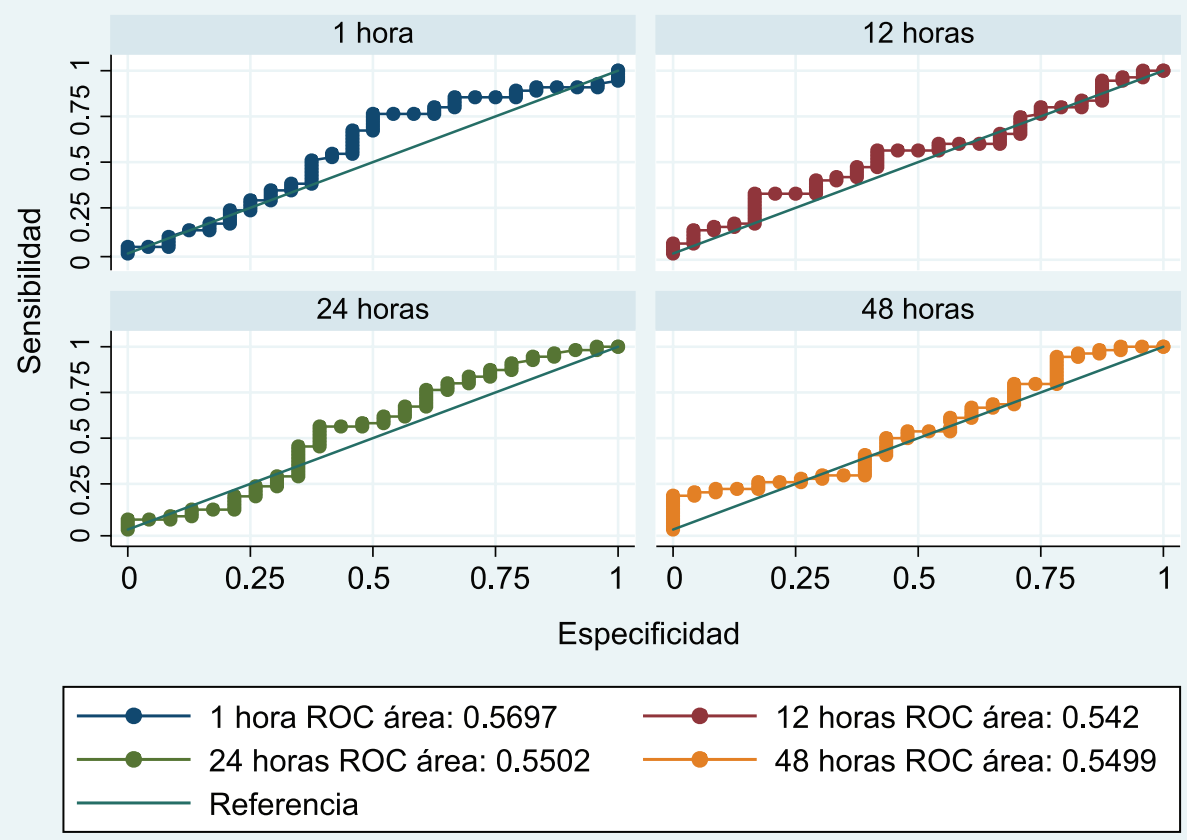

Figura 2. Curvas ROC de los valores de NGALu en las primeras 48 horas.

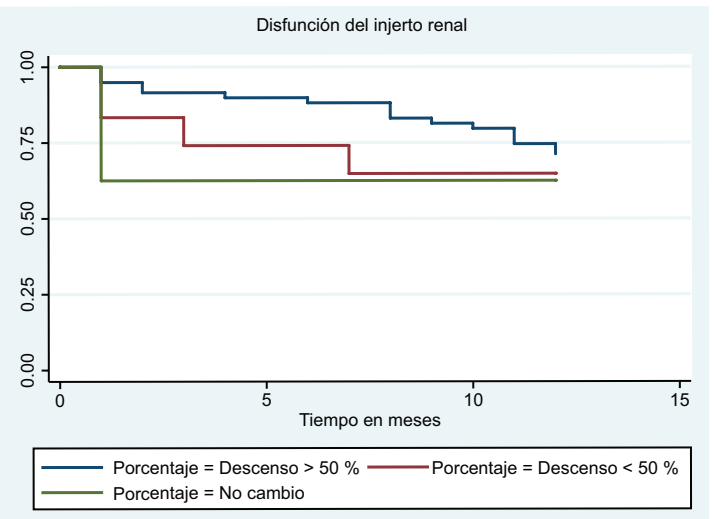

Figura 3. Curva de Kaplan-Meier para estimación de supervivencia del injerto renal según comportamiento del NGALu en las primeras 48 horas.

Tabla 2. Modelo de riesgos proporcionales de Cox para predecir disfunción del injerto renal a un año del trasplante según el comportamiento del NGALu

\begin{tabular}{lccc}
\hline & RR & IC 95 \% & p \\
\hline Descenso del NGALu* $>50$ & 1 & Referencia & \\
Descenso del NGALu* $<50$ & 1.412 & $0.474-4.205$ & 0.535 \\
Elevación del NGALu* & 1.59 & $0.466-5.439$ & 0.459 \\
\hline
\end{tabular}

${ }^{*}$ Durante las primeras 48 horas después del trasplante renal.

$\mathrm{RR}=$ razón de riesgo, $\mathrm{IC}$ = intervalo de confianza.

en los pacientes trasplantados renales sin evidencia de FRI pueden predecir la disfunción a largo plazo del injerto renal; esto podría sugerir que la lesión renal subclínica no diagnosticada por los valores de creatinina sérica pero sí por los niveles elevados de NGALu podría tener un impacto en la función del injerto renal a largo plazo, independientemente de la presencia o no de FRI y permitiría establecer estrategias de tratamiento para prevenir o limitar el daño renal antes de que sea irreversible. ${ }^{5}$

Diferentes estudios han sugerido que los valores de NGALu medidos en las primeras 48 horas después del trasplante renal predicen a largo plazo la función del injerto renal; Hall y colaboradores midieron los valores NGALu a las $0,6,12,18,24$ y 48 horas postrasplante renal de donantes fallecidos en 92 pacientes. Los pacientes con valores de NGALu mayores al percentil 66 tuvieron niveles más altos de creatinina sérica tres meses después del trasplante renal $(p=0.004))^{3}$ Este mismo grupo realizó otro estudio en 153 pacientes con trasplante renal, quienes durante el seguimiento a un año presentaron 24 pérdidas y 13 disfunciones del injerto renal. Al comparar los valores del NGALu de acuerdo con la presencia o no de disfunción del injerto renal, los valores de NGALu fueron mayores en los pacientes con este desenlace comparados con los que no (NGALu $911 \mathrm{ng} / \mathrm{mL}$ (percentiles $25-75=133-3143$ ) a diferencia de $370 \mathrm{ng} / \mathrm{mL}$ (percentiles 25-75 $=29-2381)$, respectivamente $(p=0.002)$. En el análisis de regresión logística, los valores de NGALu tomados el primer día del trasplante renal se asociaron significativamente con pobre función del 
injerto renal $(\mathrm{OR}=6$; IC $95=1.5-24) .{ }^{27}$ Choi y colaboradores en un estudio realizado en 69 pacientes con trasplante renal encontraron que los valores de NGALu fueron significativamente más altos en los pacientes con disfunción del injerto renal un año después del trasplante renal; además, en el análisis de regresión logística multivariable, los valores de NGALu al día 2 postrasplante fueron predictores independientes de pobre función del injerto $(\mathrm{OR}=1.003$; IC $95 \%=1.001-1.005) ;{ }^{18}$ sin embargo, a los dos años de seguimiento, los valores de NGALu los días 0, 2 y 6 postrasplante renal no se asociaron significativamente con pobre función del injerto. ${ }^{28}$

Fonseca y colaboradores evaluaron a 42 pacientes; en el análisis de regresión lineal encontraron que los valores de NGALu al día 7 postrasplante ajustados por el antecedente de rechazo agudo y rehospitalización se asociaron en forma independiente con los valores de creatinina sérica (coeficiente de regresión $=0.138$; IC $95 \%=0.041-0.235) .{ }^{21}$ Por el contrario, Rahizamdeh y colaboradores observaron que los valores de NGALu tomados los días 1, 3 y 7 postrasplante renal no se asociaron con disfunción renal un año después del trasplante, ${ }^{2}$ hallazgos similares a los encontrados en nuestro estudio.

Una posible explicación para las diferencias entre los resultados de estudios previos y los nuestros es el tipo de desenlace evaluado. Hall y colaboradores utilizaron una TFG $<30 \mathrm{~mL} / \mathrm{min} / 1.73 \mathrm{~m}^{2} \mathrm{sc}$ a un año del trasplante para definir la disfunción del injerto, ${ }^{3}$ Fonseca y colaboradores utilizaron como variable de desenlace los valores de creatinina sérica a un año del trasplante renal. Otra diferencia importante estriba en el tipo de población. La presencia de donantes de criterios extendidos (> 60 años, o entre 50 y 60 años con al menos dos de estos criterios: hipertensión arterial, niveles de creatinina $>1.5 \mathrm{mg} / \mathrm{dL} 0$ accidente cerebrovascular como causa de fallecimiento) se asocia con mayor riesgo de pérdida del injerto renal. ${ }^{38}$ Esto se explica porque la respuesta renal a la lesión de isquemia-reperfusión posterior el trasplante renal es un balance entre los procesos de reparación y fibrosis: ante la presencia de una lesión grave se da una fibrosis progresiva y cicatrización del injerto renal; en los casos leves o ante la presencia de injertos de adecuada calidad se logra la recuperación de las células epiteliales renales sin afectar en forma importante la función del injerto.

En nuestra cohorte no había ningún donante de criterios extendidos, todos eran jóvenes, sin comorbilidades asociadas y sin disfunción renal durante el proceso de muerte (creatinina sérica del donante antes de la extracción de $0.9 \pm 0.4 \mathrm{mg} / \mathrm{dL}$ ). Lo que podría explicar la menor frecuencia de lesión renal subclínica o la recuperación exitosa de los injertos renales ante la lesión de isquemia-reperfusión. Por el contrario, el porcentaje de donantes con criterios extendidos en los estudios de Hall y Choi fue de 18 y $30.9 \%$, 3,18 respectivamente. Así mismo, la edad de los donantes en los diferentes estudios osciló entre 42 y 51 años, ,18,21,27 mientras que la edad de los donantes de nuestra población tuvo una media de $29.6 \pm 14.18$ años.

Entre las limitaciones de nuestro estudio destaca el tamaño de la muestra y la baja proporción de pacientes con el desenlace, que sugieren que no se tuvo el poder suficiente para encontrar diferencias estadísticamente significativas y estimar el efecto de potenciales variables de confusión como el tiempo de isquemia fría, la terapia de inducción utilizada y la adherencia de los pacientes a los protocolos de tratamiento, lo cual puede influir en la función renal a corto y largo plazos.

\section{Conclusión}

En nuestra cohorte de pacientes con trasplante renal de donantes fallecidos que cumplían con criterios óptimos, los valores de NGALu en las primeras 48 horas postrasplante no se asociaron con disfunción del injerto a largo plazo. Se requieren más investigaciones para identificar el verdadero papel que cumplen los valores de NGALu en la función del injerto a largo plazo, teniendo en cuenta otras poblaciones de riesgo para $\mathrm{FRI}$ y disfunción renal.

\section{Agradecimientos}

A los Laboratorios Abbot y Novartis por la donación de kits para procesar el NGALu.

\section{Conflictos de interés}

Declaramos que ninguno de los autores tenemos conflictos de interés.

\section{Bibliografía}

1. Cohen DJ, Vella JoV. Transplantation. NephSAP. 2011;10(6).

2. Rahimzadeh N, Otukesh H, Hoseini R, Sorkhi H, Otukesh M, Hoseini S, et al. Are serum and urine neutrophil gelatinase-associated lipocalin predictive of renal graft function in short term? Pediatr Transplant. 2012;16(7):796-802.

3. Hall IE, Yarlagadda SG, Coca SG, Wang Z, Doshi M, Devarajan P, et al. IL-18 and urinary NGAL predict dialysis and graft recovery after kidney transplantation. J Am Soc Nephrol. 2010;21(1):189-197.

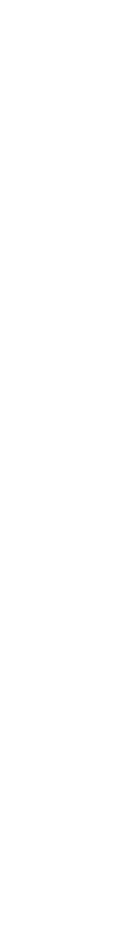


4. Alvarez S, Suazo C, Boltansky A, Ursu M, Carvajal D, Innocenti G, et al Urinary exosomes as a source of kidney dysfunction biomarker in renal transplantation. Transplant Proc. 2013;45(10):3719-3723.

5. Bataille A, Abbas S, Semoun O, Bourgeois E, Marie O, Bonnet F, et al. Plasma neutrophil gelatinase-associated lipocalin in kidney transplantation and early renal function prediction. Transplantation. 2011;92(9): 1024-1030

6. Kono H, Nakagawa K, Morita S, Shinoda K, Mizuno R, Kikuchi E, et al. Effect of a novel nuclear factor- $\mathrm{B}$ activation inhibitor on renal ischemia-reperfusion injury. Transplantation. 2013;96(10):863-870.

7. Parikh CR, Jani A, Mishra J, Ma Q, Kelly C, Barasch J, et al. Urine NGAL and IL-18 are predictive biomarkers for delayed graft function following kidney transplantation. Am J Transplant. 2006;6(7):1639-1645.

8. Haase M, Bellomo R, Devarajan P, Schlattmann P, Haase-Fielitz A NGAL Meta-analysis Investigator Group. Accuracy of neutrophil gelatinase-associated lipocalin (NGAL) in diagnosis and prognosis in acute kidney injury: a systematic review and meta-analysis. Am J Kidney Dis. 2009;54(6):1012-1024

9. Lin M, Li L, Li L, Pokhrel G, Qi G, Rong R, et al. The protective effect of baicalin against renal ischemia-reperfusion injury through inhibition of inflammation and apoptosis. BMC Complement Altern Med. 2014;14(1):19.

10. Perico N, Cattaneo D, Sayegh M, Remuzzi G. Delayed graft function in kidney transplantation. Lancet. 2004;364:1814-1827.

11. Yarlagadda SG, Coca SG, Garg AX, Doshi M, Poggio E, Marcus RJ, et al. Marked variation in the definition and diagnosis of delayed graft function: a systematic review. Nephrol Dial Transplant. 2008;23(9): 2995-3003.

12. Devarajan P. NGAL in acute kidney injury: from Serendipity to utility. Am J Kidney Dis. 2008:52(3):395-399.

13. Adiyanti SS, Loho T. Acute kidney injury (AKI) biomarker. Acta Med Indones. 2012;44(3):246-255.

14. Hall IE, Doshi MD, Poggio ED, Parikh CR. A comparison of alternative serum biomarkers with creatinine for predicting allograft function after kidney transplantation. Transplantation. 2011;91(1):48-56.

15. Heyne N, Kemmner S, Schneider C, Nadalin S, Königsrainer A Häring HU. Urinary neutrophil gelatinase-associated lipocalin accurately detects acute allograft rejection among other causes of acute kidney injury in renal allograft recipients. Transplantation. 2012;93(12):1252-1257.

16. Lee EY, Kim MS, Park Y, Kim HS. Serum neutrophil gelatinase-associated lipocalin and interleukin-18 as predictive biomarkers for delayed graft function after kidney transplantation. J Clin Lab Anal. 2012;26(4):295-301.

17. Bolignano D, Donato V, Coppolino G, Campo S, Buerni A, Lacquaniti A et al. Neutrophil gelatinase-associated lipocalin (NGAL) as a marker of kidney damage. Am J Kidney Dis. 2008;52(3):595-605

18. Choi HM, Park KT, Lee JW, Cho E, Jo SK, Cho WY, et al. Urine neutrophil gelatinase-associated lipocalin predicts graft outcome up to 1 year after kidney transplantation. Transplant Proc. 2013;45(1):122-128.

19. Rostami Z, Nikpoor M, Einollahi B. Urinary neutrophil gelatinase associated lipocalin (NGAL) for early diagnosis of acute kidney injury in rena transplant recipients. Nephrourol Mon. 2013:5(2):745-752.

20. Kanter J, Beltran S, Molina D, Vallecillo J, Sancho A, Gavela E, et al Urinary neutrophil gelatinase-associated lipocalin after kidney transplantation: is it a good biomarker to assess delayed graft function? Transplant Proc. 2013:45(4):1368-1370.

21. Fonseca I, Oliveira JC, Almeida M, Cruz M, Malho A, Martins LS, et al. Neutrophil gelatinase-associated lipocalin in kidney transplantation is an early marker of graft dysfunction and is associated with one-year renal function. J Transplant. 2013;2013:650123.
22. Hollmen ME, Kyllönen LE, Inkinen KA, Lalla MLT, Salmela KT. Urine neutrophil gelatinase-associated lipocalin is a marker of graft recovery after kidney transplantation. Kidney Int. 2011;79(1):89-98.

23. Lebkowska U, Malyszko J, Lebkowska A, Koc-Zorawska E, Lebkowski W, Malyszko JS, et al. Neutrophil gelatinase-associated lipocalin and cystatin $\mathrm{C}$ could predict renal outcome in patients undergoing kidney allograft transplantation: a prospective study. Transplant Proc. 2009;41(1): 154-157.

24. Kaufeld JK, Gwinner W, Scheffner I, Haller HG, Schiffer M. Urinary NGAL ratio is not a sensitive biomarker for monitoring acute tubular injury in kidney transplant patients: NGAL and ATI in renal transplant patients. J Transplant. 2012;2012:563404.

25. Vanmassenhove J, Vanholder R, Nagler E, Van-Biesen W. Urinary and serum biomarkers for the diagnosis of acute kidney injury : an in-depth review of the literature. Nephrol Dial Transplant. 2013;28(10):254-273.

26. Field M, Lowe D, Cobbold M, Higgins R, Briggs D, Inston N, et al. The use of NGAL and IP-10 in the prediction of early acute rejection in highly sensitized patients following HLA-incompatible renal transplantation. Transpl Int. 2014;27(4):362-370.

27. Hall IE, Doshi MD, Reese PP, Marcus RJ, Thiessen-Philbrook H, Parikh CR. Association between peritransplant kidney injury biomarkers and 1-year allograft outcomes. Clin J Am Soc Nephrol. 2012;7(8): 1224-1233.

28. Yang J, Choi HM, Seo MY, Lee JY, Kim K, Jun H, et al. Urine liver-type fatty acid-binding protein predicts graft outcome up to 2 years after kidney ransplantation. Transplant Proc. 2014:46(2):376-380.

29. Nieto-Ríos JF, Serna-Higuita LM, Ocampo C, Aristizabal A, Vélez C, Vanegas JJ, et al. NGAL como predictor temprano de función retardada del injerto renal. Biomedica. 2016;36(2):213-219.

30. Shaffi K, Uhlig K, Perrone RD, Ruthazer R, Rule A, Lieske JC, et al. Performance of creatinine-based GFR estimating equations in solid-organ transplant recipients. Am J Kidney Dis. 2014;63(6):1007-1018.

31. Díaz $P$, Fernández $P$. Cálculo del tamaño muestral para la determinación de factores pronósticos. Cad Aten Primaria. 2002;9:30-33.

32. Halloran PF, Aprile MA, Farewell V, Ludwin D, Smith EK, Tsai SY, et al. Early function as the principal correlate of graft survival. A multivariate analysis of 200 cadaveric renal transplants treated with a protocol incorporating antilymphocyte globulin and cyclosporine. Transplantation. 1988;46.(2):223-228.

33. Johnston O, O'kelly P, Spencer S, Donohoe J, Walshe JJ, Little DM, et al. Reduced graft function (with or without dialysis) vs immediate graft function: a comparison of long-term renal allograft survival. Nephrol Dial Transpl. 2006;21(8):2270-2274.

34. Yarlagadda SG, Coca SG, Formica RN, Poggio ED, Parikh CR. Association between delayed graft function and allograft and patient survival: a systematic review and meta-analysis. Nephrol Dial Transplant. 2009;24(3):1039-1047.

35. Ponticelli C. Ischaemia-reperfusion injury: a major protagonist in kidney transplantation. Nephrol Dial Transplant. 201429(6):1134-1140.

36. Mishra J, Ma Q, Kelly C, Mitsnefes M, Mori K, Barasch J, et al. Kidney NGAL is a novel early marker of acute injury following transplantation. Pediatr Nephrol. 2006;21(6):856-863.

37. Ramirez-Sandoval JC, Herrington W, Morales-Buenrostro LE. Neutrophil gelatinase-associated lipocalin in kidney transplantation: a review. Transplant Rev. 2016;29(3):139-144.

38. Melilli E, Bestard O, Cruzado JM, Zorita IN, Grinyó JM, Castelao AM. Trasplante de riñones con criterios expandidos : manejo y resultados a largo plazo. Nefrologia. 2011;2(5):98-104. 